\title{
Nano-reinforcement in sustainable polymer composites
}

Muhammad Khusairy Bin Bakri ${ }^{1}$, Md. Rezaur Rahman', Siti Noor Linda Taib ${ }^{7}$, Perry Law Nyuk Khui ${ }^{1}$, Akshay Kakar ${ }^{2}$ and Elammaran Jayamani ${ }^{3}$

${ }^{1}$ Faculty of Engineering, Universiti Malaysia Sarawak, Jalan Datuk Mohammad Musa, Kota Samarahan, Malaysia, ${ }^{2}$ Department of Materials Science and Engineering, University of Houston, Houston, TX, United States, ${ }^{3}$ Faculty of Engineering, Computing and Science, Swinburne University of Technology, Sarawak Campus, Kuching, Malaysia

\subsection{Introduction}

The advancement of the materials and their development has always been a crucial part of human development. It was therefore important to categorize materials as a reference to their stages of development; that is, the first "Stone" Age, followed by the "Bronze" Age, "Iron" age, and in the modern time, namely, "plastic" age, "silicon" age, and "nanomaterial" age (Malik et al., 2019). It is estimated that around $70 \%$ of all technical innovations are attributed indirectly and directly based on the materials' properties, whereas in the macroscopic world, the solutions are being explored on how to incorporate them with other components (Malik et al., 2019).

It is also reasonable to mention that we are in the "composite" age. Practically, all technological sectors and branches of industry show that nanocarbons display unique properties to innovate. Most of the innovation in nano-reinforcement materials are generally to improve the quality of our lives and used as cutting-edge technology, due to its potential in saving energy, conserving resources, and reducing environmental pollution (Malik et al., 2019).

Extensive research has been done in the area of nanocarbon materials over 30 years ago since the discovery of fullerenes. It was boosted extensively when carbon nanotubes (CNTs) were discovered and then expanded with the advent of graphene and upgraded to the area of two-dimensional materials (Malik et al., 2019). Due to the diverse research area, the applications are highlighted especially for its promising performance in coatings, biomedicine, biosensors, energy transfer composites, advanced nanocarbon materials, and diagnostics.

In composite developments, decreasing size from macro- to meso- and micro- to nano-scopes is preferred and used as reinforcement materials (Fiedler et al., 2006). The development directly affected and is related to the development of methodological variations, concerning the investigation and experimental setup and methods. Most of it is not valid anymore for nanofiller as the identified conventional theories 